\title{
Pore fabric shape anisotropy in porous sandstones and its relation to elastic wave velocity and permeability anisotropy under hydrostatic pressure.
}

\author{
Philip M Benson ${ }^{1}$, Philip G Meredith ${ }^{1}$, Ellen S Platzman ${ }^{1}$ and Roy E White ${ }^{2}$ \\ ${ }^{1}$ Department of Earth Sciences, University College London, Gower Street, London \\ WC1E6BT, UK (p.benson@ucl.ac.uk). \\ ${ }^{2}$ School of Earth Sciences, Birkbeck College, Malet Street, LONDON, WC1E 7HX, \\ UK.
}

\begin{abstract}
Most crustal rocks are anisotropic. Anisotropy may result due to depositional pore fabric development, diagenesis, and preferred directions of microcracks and crystallographic alignments. To further the understanding between some of these mechanisms and commonly measured rock physics properties (ultrasonic velocity, permeability and porosity), we developed a novel apparatus capable of measuring these parameters simultaneously at hydrostatic pressures up to $100 \mathrm{MPa}$. First, we determine the average 3-D void space shape and orientation using the methods of Anisotropy of Magnetic Susceptibility and acoustic wave velocity (P-wave and Swave) to identify the principal anisotropy axes under ambient laboratory conditions. This directional anisotropy data is then used to guide experiments under hydrostatic pressure, where permeability, porosity and ultrasonic velocity are measured simultaneously from 5 to $90 \mathrm{MPa}$. We describe the use of these methods in an comparative study using two sandstones (Bentheim and Crab Orchard) to investigate the relationship between permeability, acoustic anisotropy, and pore fabric geometry. We find the structural anisotropy formed by the void space is well described by velocity anisotropy in both cases. At ambient (room) pressure, Crab Orchard sandstone has a velocity anisotropy of $19.1 \%$ and $7.6 \%$ (for P-waves and S-waves respectively). In contrast Bentheim sandstone possesses a weaker anisotropy of $4.7 \%$ and $3.0 \%$ for P-wave and S-wave velocity respectively. Under hydrostatic pressure, the acoustic anisotropy of Crab Orchard sandstone decreases rapidly from 3\% and 7\% at $5 \mathrm{MPa}$ (P-wave and $\mathrm{S}$-wave) to $1.5 \%$ and $1 \%$ respectively at effective pressures over 40MPa; while for Bentheim sandstone the decrease is considerably less.
\end{abstract}


Permeability of Crab Orchard sandstone is $125 \times 10^{-18} \mathrm{~m}^{2}$, decreasing rapidly as effective pressure increases. Fluid flow is highly dependent on direction with respect to pore fabric; permeability parallel to bedding is approximately twice that normal to bedding. In contrast, permeability of Bentheim sandstone is $0.86 \times 10^{-12} \mathrm{~m}^{2}$, and varies little with effective pressure or coring direction. We relate many of our measurements made under hydrostatic pressure to the contrasting pore fabric between the two rock types, and infer that a critical pressure is required for the initiation of crack closure, as expected from many models cited in the literature.

\section{Introduction}

Porosity is a ubiquitous feature of sedimentary rock. However, the processes responsible for porosity formation are diverse; ranging from depositional processes such as sorting and grain alignment, through diagenetic processes such as compaction and cementation, to deformational processes such as microcracking and pressure solution. The porosity that evolves from the superposition of these processes over time may therefore have a complex geometry or fabric. In particular, many of these processes, such as grain sorting, compaction and microcracking, have an inherent directionality which may lead to anisotropy of the void space. This is important, since the void space geometry in porous rock is one of the key controls on other mechanical and physical properties. Clearly, therefore, the traditional measurement of porosity as a volume-averaged, scalar quantity is not capable of providing information about the shape and geometry of the void spaces which constitute that porosity.

A common way of addressing this problem is to use elastic wave velocity measurement to estimate pore space shape and orientation $[1,2]$. Wave velocities provide a reasonable proxy for porosity since they are very sensitive to the presence of pore volume within a solid matrix. Velocity measurement is relatively straightforward and, if done in multiple orientations, can be used to estimate pore space anisotropy. However, a number of problems exist with this approach. For example, it is widely accepted that high and low aspect ratio porosity (such as equant pores and thin cracks respectively) can exert disproportionate influences on wave velocities $[1,2,3]$, and especially on their anisotropy. Also, wave velocities are 
affected by all the void space and not just the connected porosity. Furthermore, factors other than the void space geometry may affect velocity anisotropy. For example, the rock matrix may exhibit anisotropy through an aligned grain fabric or from a crystallographically preferred orientation within the grains themselves. In situations where the anisotropy is evident, the elastic anisotropy parameters are often calculated using three orthogonal cores [4], with a fourth core prepared at an angle to these (most commonly at $45^{\circ}$, for mathematical simplicity). An alternative method is to make multiple radial measurements around the circumference of each core of a three orthogonal core set. This allows an approximate velocity ellipsoid to be calculated [3,5], from which the principal anisotropy axes can be determined regardless of the orientation of the axes of the core set.

Alternatively, void space geometry can be determined using the method of anisotropy of magnetic susceptibility (AMS). AMS has traditionally been used to measure the magnetic anisotropy of a dry rock matrix (mAMS) [6]. Used in this manner it has become a common tool for measuring sedimentary grain fabrics, and hence for inferring depositional process and palaeocurrent directions [6]. However, this tells us nothing about the void space. To determine void space anisotropy using this technique, we saturate the pore space with a highly susceptible magnetic ferrofluid, thus providing it with an artificial magnetic susceptibility. Assuming that any magnetic minerals can be removed or neglected, measurement of the pore space AMS, which we term pAMS, now reflects the bulk pore space volume, shape and alignment $[3,7,8]$. The technique has several advantages over other methods. Firstly, AMS (mAMS and pAMS) is a second rank tensor, and can therefore be described by an ellipsoid. Secondly, the 3D anisotropy ellipsoid can be fully determined from measurements on a single core. Anisotropy can therefore be quickly determined, regardless of the orientation of the core axis with respect to the principal anisotropy directions. In most cases, the pore space anisotropy can be determined independently of the matrix anisotropy. Note, however, that pAMS measures the anisotropy of the connected pore space that is accessible to the ferrofluid rather than the total porosity. For this reason, past work $[8,3]$ has concentrated on demonstrating the strong correlation between pAMS and permeability anisotropy, since there is no direct, general relation between permeability and scalar porosity [9] but permeability 
anisotropy is likely to be governed by the geometry and connectivity of the elements that make up the pore space which is well described by pAMS.

While each technique has its merits, we consider that, since they measure different but complementary properties, they are more powerful when used together. Consequently, here we report measurements of acoustic velocity anisotropy and pAMS on two different porous sandstones under ambient laboratory conditions. The principal anisotropy directions derived from these data are then used to guide a detailed experimental programme in which $\mathrm{P}$-wave and S-wave ultrasonic velocities, permeability and porosity are measured simultaneously at effective pressures up to $90 \mathrm{MPa}$. Analysis of the changes in velocity and permeability, and their anisotropy, provides a detailed picture of how the pore fabric responds to the hydrostatic pressure acting on the rocks.

\section{Test materials}

Two rock types were chosen for this study; Bentheim sandstone from the Lower Saxony Basin, north-west Germany, and Crab Orchard sandstone from the Cumberland Plateau, Tennessee, USA. Scanning electron micrographs of freshly broken surfaces of the two rocks (Figure 1) illustrate their very different microstructures. Bentheim sandstone (BHS) is an off-white sandstone with a porosity of $23.7 \pm 0.7 \%$. Mineralogically it is composed of $>95 \%$ quartz grains held together by a siliceous cement, with feldspar and mica constituting less than $1 \%$ and clay minerals about 3\%. Grains are well-sorted, sub-rounded and have a mean size of approximately $0.2 \mathrm{~mm}$. No obvious layering or fabric can be seen in hand specimen. By contrast, Crab Orchard sandstone (COS) shows clear, cross-bedded layering on the millimetre scale in hand specimen. Grains are generally subhedral to sub-rounded, exhibit no preferred alignment, and have a mean size of approximately $0.2 \mathrm{~mm}$. Porosity is $4.5 \pm 0.5 \%$. Compositionally the rock is $85 \%$ quartz with significant feldspar and some lithics. A cement of sericitic clay and mica is abundant, and this appears to have destroyed much of the original porosity. Cementation is thought to 
have occurred early on in diagenesis, as nearly all grains show triple junctions, with few point contacts.

All core samples used in this study were taken from single blocks of each material in order to minimise sample variability. Cores were taken along three orthogonal directions, as illustrated in Figure 2. For COS, the ' $Z$ ' axis is taken as normal to the observed bedding. For BHS, where there is no visible fabric in hand specimen, the ' $Z$ ' axis is taken as normal to the large scale bedding observed in the quarry.

\section{Anisotropy measurements at ambient pressure}

\subsection{Pore space anisotropy}

Pore space anisotropy, defined as the relative shape and alignment of the average porosity ellipsoid, was determined from anisotropy of magnetic susceptibility measurements of samples saturated with magnetic ferrofluid (pAMS), using the method described by Benson et al [3]. Sample dimensions were $25 \mathrm{~mm}$ diameter by approximately $22 \mathrm{~mm}$ in length. Principal pAMS directions are determined by measuring the susceptibility in 15 separate directions, and fitting these data to an ellipsoid [3,7]. In our experiments, we found that the magnitude of the susceptibility of ferrofluid impregnated samples was some 3 orders of magnitude greater than the natural matrix susceptibility. The pAMS thus reflects the average shape and orientation of the total connected pore space, with the pAMS anisotropy parameter ' $\mathrm{P}$ ' being defined in terms of the lengths of these principal axes: $\mathrm{P}_{\mathrm{AMS}}=\left(\mathrm{k}_{\max }-\mathrm{k}_{\min }\right) / \mathrm{k}_{\text {mean }}$, where $k_{x}$ is the susceptibility along principal axis ' $\mathrm{x}$ '.

\subsection{Elastic wave velocity anisotropy}

Elastic wave velocity measurements were made on core samples of $38 \mathrm{~mm}$ diameter by $40 \mathrm{~mm}$ length, with three cores taken in each of the three orthogonal directions indicated in Figure 2, and for each rock type. P-wave velocity (Vp) and Swave velocity (Vs) was then measured across the diameter of the cores, in $10^{\circ}$ radial 
increments, using the time of flight method $[3,10]$. Measurements were made using transducers with a central frequency of $1 \mathrm{MHz}$, so that the wavelength corresponded to approximately 10 grain diameters and the sample size greater than 10 wavelengths. This ensured that scattering effects were minimized, and that the acoustic wave measured average rock properties.

By using nine cores and 36 radial velocity measurements per core, we are able to fit the velocity data to an ellipsoid [3,5], which we term an ellipsoidal velocity model (EVM). Since elastic anisotropy is a fourth rank tensor, this is clearly an approximation in general terms. To evaluate the goodness of fit between this approximation and the data, the forward solution to the model was performed. This reveals that the error in the ellipsoidal fit is less than or equal to the velocity measurement accuracy, of approximately $2 \%$. However, the use of the EVM has two important advantages; (1) it enables us to determine principal anisotropy directions in three dimensions, regardless of the orientations in which the cores were taken $[3,5]$, and (2) it enables a direct and simple comparison between wave velocity and pAMS measurements of anisotropy. For elastic wave velocity, we use the anisotropy parameter ' $\mathrm{P}_{\mathrm{v}}$ ', defined in terms of the lengths of the principal axes generated from the EVM procedure: $\mathrm{P}_{\mathrm{v}}=\left(\mathrm{v}_{\max }-\mathrm{v}_{\min }\right) / \mathrm{v}_{\text {mean }}$, where $\mathrm{v}_{\mathrm{x}}$ is the velocity in the direction of principal axis ' $\mathrm{x}$ '.

\section{Measurements under hydrostatic pressure}

The AMS and wave velocity measurements described above were all made at ambient pressure to characterise the initial pore fabric of the two sandstones. In order to extend the measurements to more realistic crustal conditions, a new, high pressure permeameter/volumometer with a simultaneous ultrasonic wave velocity measurement capability was developed. This is essentially a re-development of an earlier apparatus described by Jones \& Meredith [11], and comprises a $300 \mathrm{MPa}$ hydrostatic pressure vessel equipped with dual $70 \mathrm{MPa}$ servo-controlled pore fluid intensifiers for permeability and porosity measurement (Figure 3). Rubber-jacketed samples are housed inside the pressure vessel between two steel end-caps. The endcaps perform two main functions. Firstly, they contain ports to allow pore fluid to be 
introduced and distributed to one end of the sample, and to be received and expelled at the other end. Secondly, they house piezoelectric P-wave and S-wave transducers for the measurement of axial elastic wave velocities. A diagram of the sample assembly is shown in Figure 4. All measurements were made on samples $38 \mathrm{~mm}$ in diameter by $40 \mathrm{~mm}$ long, cored parallel and normal to the principal anisotropy axes as determined from the ambient pressure measurements described above.

Each servo-controlled intensifier independently provides up to $70 \mathrm{MPa}$ of pore pressure to either end of the sample. The intensifiers are fitted with integral displacement transducers which monitor the position of the high-pressure pistons, thus enabling the intensifiers also to be used as volumometers. For permeability measurements, the upstream and downstream intensifiers were set to slightly different control pressures in order to maintain a small, constant pressure difference across the sample. The actual pressure difference used depends on the rock being tested, but is typically in the range 0.2 and $0.5 \mathrm{MPa}$ for a minimum mean pore fluid pressure of 2.5 $\mathrm{MPa}$. Once steady-state flow is established, permeability can be determined from the rate of fluid flow, the pressure gradient, and the sample dimensions via direct application of Darcy's law. To minimise any temperature-induced pressure fluctuations, the equipment is located within a temperature-controlled laboratory, and a thermocouple is used to continuously monitor the temperature inside the pressure vessel next to the sample assembly during each experiment. Using this system, permeability measurements were made on both rock types at $5 \mathrm{MPa}$ intervals for simple effective pressures from 5 to $20 \mathrm{MPa}$, and at $10 \mathrm{MPa}$ intervals for effective pressures from 20 to $90 \mathrm{MPa}$. In this study, we use the simple effective pressure law [12] $\mathrm{P}_{\text {eff }}=\mathrm{P}_{\mathrm{c}}-\alpha \mathrm{P}_{\mathrm{p}}$, with $\alpha=1$. Effective pressure is varied during our experimental tests by holding the mean pore pressure constant at $2.5 \mathrm{MPa}$, and increasing the confining pressure stepwise until the desired values of $\mathrm{P}_{\text {eff }}$ is achieved.

Changes in sample porosity with increasing effective pressure were made simultaneously via the following method. Prior to increasing the effective pressure between permeability measurements, the downstream reservoir isolation valve (Figure 3 - intensifier ' $A$ ') was closed and the pressure in the upstream reservoir and sample allowed to equilibrate. After equilibration, the effective pressure is increased and the displacement of the upstream intensifier piston monitored. This displacement directly 
measures the volume of water expelled from the sample due to the closure of cracks and pores, i.e. the change in porosity due to the increase in effective pressure.

Simultaneous measurement of axial P-wave and S-wave velocity were made at each of the effective pressures for which permeability and porosity measurements were made. The two sample end-caps each house two $1 \mathrm{MHz}$ piezoelectric transducers, one compressional $(\mathrm{P})$ mode and one shear $(\mathrm{S})$ mode, bonded to the back of the pore fluid distribution plate and isolated from the confining and pore pressures behind a high pressure seal (Figure 4). All measurements were made using the timeof-flight technique, and the same instrumentation (pulser, amplifier and digital storage oscilloscope) as for the ambient pressure measurements. Average error between subsequent measurements in permeability, porosity and acoustic wave velocity is estimated at $2 \%, 1 \%$ and $0.5 \%$ respectively.

\section{Results}

\subsection{Measurements at ambient pressure}

Raw ultrasonic wave velocity results made under ambient conditions are presented in Figure 5. When these data are processed via the EVM method, BHS exhibits a weak anisotropy of $4.7 \%$ and $3.0 \%$, for $\mathrm{Vp}$ and $\mathrm{Vs}$ respectively. In contrast, COS exhibits a significant Vp and Vs anisotropy of $19.1 \%$ and $7.6 \%$ respectively (table 1). Since the cores are taken in three orthogonal directions, one radial direction on each core has a co-incident direction on another core. For the un-polarised P-waves this provides a check on the measurements. For example, it can be seen in Figure 5 that the velocities coincide within measurement error at orientations of $90^{\circ}$ and $270^{\circ}$.

Results from pAMS measurements reveal a very similar pattern of void space anisotropy (Table 1). Crab Orchard sandstone exhibits a prominent anisotropy, with the minimum principal susceptibility axis (Figure 6) lying sub-parallel to the $\mathrm{Z}$ axis, i.e. sub-normal to the observed cross-bedding, and with both intermediate and maximum principal pAMS axes distributed around a plane sub-parallel to the crossbedding. These pAMS results are in close agreement with principal anisotropy 
directions calculated from the P-wave and S-wave velocity data also reported in Table 2 and Figure 6.

Similar results are also observed for Bentheim sandstone (Figure 6 and Table 2). Despite the lack of observable bedding in hand specimen, a pAMS-inferred void space minimum is observed approximately parallel to the $\mathrm{Z}$ axis, i.e. sub-normal to the large-scale bedding observed in the quarry. Again, intermediate and maximum principal pAMS axes are distributed around a plane at approximately $90^{\circ}$ to the minimum direction; sub-parallel to the large-scale bedding. Once again, the same pattern is seen in the principal orientations calculated from the wave velocity data and presented in Figure 6.

\subsection{Measurements under hydrostatic pressure}

Following characterization under ambient conditions, a series of measurements were made at hydrostatic effective pressures from 5 to $90 \mathrm{MPa}$ on a sub-set of the $38 \mathrm{~mm}$ diameter samples used for the ambient velocity measurements. Permeability, porosity, and axial $\mathrm{Vp}$ and Vs were measured simultaneously at each incremental increase in effective pressure on cores with axes in each of the $\mathrm{X}, \mathrm{Y}$ and $\mathrm{Z}$ directions. For simplicity, we refer to measurements made on a sample cored in a particular orientation with a suffix; for example, the axial $\mathrm{P}$-wave velocity in the $\mathrm{Z}$ direction is written as $\mathrm{Vp}(\mathrm{Z})$.

For both rocks, Vp increases and the porosity decreases with increasing effective pressure, as expected (Figure 7). For BHS, Vp increases at a lower average rate and the relationship between effective pressure and velocity appears to become linear above about $50 \mathrm{MPa}$. In contrast, for COS Vp continues to increase up to the maximum effective pressure attained, at a continuously decreasing rate towards higher pressure. $\mathrm{Vp}(\mathrm{Z})$ is significantly lower than either $\mathrm{Vp}(\mathrm{X})$ or $\mathrm{Vp}(\mathrm{Y})$, which essentially have the same value. Since this observation occurs many times, for simplicity we plot the average of $\mathrm{Vp}(\mathrm{X})$ and $\mathrm{Vp}(\mathrm{Y})$, which we will refer to as $\mathrm{Vp}(\mathrm{X}) / \mathrm{Vp}(\mathrm{Y})$ for the rest of this paper; with a similar notation used for S-wave velocity and permeability. A similar overall trend is seen for Vs (Figure 7). However, for $\operatorname{COS}$ there is a large initial difference between $\operatorname{Vs}(\mathrm{Z})$ and $\operatorname{Vs}(\mathrm{X}) / \mathrm{Vs}(\mathrm{Y})$ that 
decreases rapidly as pressure is increased to about $30 \mathrm{MPa}$. Above this pressure, all the velocities follow a similar trend. Figure 8 shows the variation in velocity anisotropy for both rocks as a function of effective pressure. For BHS, there is only a very small initial decrease in Vp anisotropy, and above about $10 \mathrm{MPa}$ the anisotropy remains essentially constant up to $90 \mathrm{MPa}$. Vs anisotropy, however, shows a small, but distinct, decrease which continues even to high effective pressure. In contrast, COS shows a much more obvious decrease in acoustic anisotropy (both Vp and Vs) as effective pressure is increased from 5 to $30 \mathrm{MPa}$, consistent with the velocity variations noted above. The decrease is particularly marked for Vs, where the anisotropy decreases from approximately $8 \%$ to $1 \%$.

The porosity over the same effective pressure range, measured contemporaneously, is also plotted on Figure 7. This is calculated by measuring the porosity change with pressure at each stepwise effective pressure increment, and the known porosity at the start of each test. In general, the change in porosity mirrors the change in velocities, and decreases with increasing effective pressure. However, there are marked differences between the porosity changes in the two rocks. COS has an ambient pressure porosity of only 0.044 , and this decreases by some $17 \%$ over the pressure range 5 to $90 \mathrm{MPa}$. In contrast, BHS has a much higher initial porosity (0.229), but this only decreases by a little over $3 \%$ over the same pressure range.

Permeability of the two rock types also differs greatly, in terms of absolute value, anisotropy, and variation with effective pressure. These differences are illustrated in figures 9 and 10. The average permeability (k) of high-porosity BHS at $5 \mathrm{MPa}$ effective pressure is approximately $0.86 \times 10^{-12} \mathrm{~m}^{2}$. This value varies little with either orientation or change in effective pressure. For the lower porosity COS, the permeability at $5 \mathrm{MPa}$ is more than three orders of magnitude lower. It is also highly anisotropic, with the permeability parallel to bedding $(\mathrm{k}(\mathrm{X}) / \mathrm{k}(\mathrm{Y}))$ having a value of $125 \times 10^{-18} \mathrm{~m}^{2}$, more than twice that normal to bedding $(\mathrm{k}(\mathrm{Z}))$. In contrast with BHS, both $\mathrm{k}(\mathrm{X}) / \mathrm{k}(\mathrm{Y})$ and $\mathrm{k}(\mathrm{Z})$ for COS decrease by more than an order of magnitude as effective pressure is increased from 5 to $90 \mathrm{MPa}$. Significantly, however, the permeability anisotropy varies little over the pressure range for either rock. 


\section{Discussion}

Results of measurements made under ambient conditions show that COS is highly anisotropic, with a P-wave anisotropy of $19.1 \%$ and S-wave anisotropy of $7.6 \%$. By comparison, BHS exhibits a significantly lower anisotropy of $4.7 \%$ and $3.0 \%$ for P-wave and S-wave velocity respectively (Figure 5). Measurements of the pore fabrics, using pAMS, are consistent with this observation. The void space of COS shows a volume averaged (pAMS) anisotropy of 3.8\%, compared with $1.5 \%$ for BHS (Table 1). Both pAMS and ultrasonic wave velocity techniques have also been used to determine principal anisotropy axes through fitting the directional data to ellipsoids. However, the validity of the EVM approximation used for fitting acoustic velocity data to ellipsoids depends upon the symmetry of the elastic tensor. By examining data presented in this paper, we ultimately find that we are dealing with rocks exhibiting an approximate planar transverse isotropy. Theoretically, this form of anisotropy possesses five independent elastic constants, and so the approximation to a second order ellipsoid with three elastic constants is good. In addition, the fact that the velocity anisotropy agrees well to this ellipsoidal fit suggests that we are dealing with rocks in which the three primary parameters used may accurately describe the five constants which are, strictly, needed.

Ultrasonic P-wave velocity results provide the most highly clustered data (Figure 6). Generally, directional data for the principal axes of Vp and Vs lie within the same quadrant, but commonly outside the reported statistical error. In particular, it is difficult to separate the S-wave maximum and intermediate directions for COS. This is likely to be due to the complex interaction between the polarized S-wave and the highly anisotropic pore fabric [3]. We also note that fitting the velocity data to an ellipsoid represents an approximation, since the elastic anisotropy can only be fully described by a fourth rank tensor [3,7]. Principal pAMS axis orientations show a high degree of correlation with the principal velocity anisotropy directions, supporting the deduction that the internal pore fabric is the dominant factor controlling the anisotropy of both rocks. This appears to be true for both COS, where the bedding is clearly visible, and for BHS, where no visible structure can be seen at the sample scale. However, due to the characteristics of the pAMS technique, which measures 
only the anisotropy of the connected void space, we consider that it is more likely to be an accurate predictor of fluid flow anisotropy $[3,8]$ than velocity anisotropy.

As noted earlier, the mean initial permeability of Bentheim sandstone under a hydrostatic pressure of $5 \mathrm{MPa}$ is $0.86 \times 10^{-12} \mathrm{~m}^{2}$; this value barely changes either with increase in effective pressure or with orientation. This is in spite of the fact that both wave velocity and pAMS measurements indicate a low, but significant, anisotropy. This suggests both that the anisotropy is not sufficiently high compared with the porosity to influence fluid flow, and that the pore connectivity is not greatly altered by elevated pressure. The SEM micrographs in Figure 1 support this interpretation, since we observe a highly connected set of relatively equant pores with little evidence of any microcracks or cement. This observation is also consistent with the results of Jones \& Meredith [11], who reported no significant change in permeability with increasing effective pressure for a tuff with predominantly equant porosity.

These observations are in stark contrast with those for Crab Orchard sandstone, where permeability changes markedly, both as a function of pressure and flow direction. The directions of maximum and minimum flow are in good agreement with the principal directions indicated by pAMS, Vp and Vs anisotropy. Initial permeability parallel to crossbedding $(\mathrm{k}(\mathrm{X}) / \mathrm{k}(\mathrm{Y}))$ is more than twice that normal to crossbedding $(\mathrm{k}(\mathrm{Z}))$, with an anisotropy of approximately $90 \%$; far higher than for either pAMS or velocity anisotropy. Also, while the permeability decreases by more than an order of magnitude as effective pressure is increased from 5 to $90 \mathrm{MPa}$, the permeability anisotropy remains approximately constant throughout (Figure 9), suggesting that the mechanism responsible for the reduction in permeability is acting to reduce fluid flow magnitude by the same relative amount independently of flow direction. Again, this observation is in agreement with previously reported results [11] for a rock microstructure comprising a mixture of microcracks and open pores. However, it is marked contrast to results for rocks with a predominantly planar fabric, e.g. shales, where increasing effective pressure reduces the fluid flow parallel to the fabric much more than that normal to it, thus reducing the anisotropy to close to zero [11]. 
During each test, simultaneous changes in P-wave and S-wave velocities and porosity were monitored as a function of effective pressure (Figure 7). For COS, marked changes are observed in porosity, velocity, and velocity anisotropy. A porosity decrease of $17 \%$ over the pressure range 5 to $90 \mathrm{MPa}$ results in increases in P-wave velocities of between $16 \%$ and $17 \%$ (for $\mathrm{Vp}(\mathrm{X}) / \mathrm{Vp}(\mathrm{Y})$ axis and $\mathrm{Vp}(\mathrm{Z})$ respectively). S-wave velocities increase even more, with $\mathrm{Vs}(\mathrm{X}) / \mathrm{Vs}(\mathrm{Y})$ rising by $21 \%$ and $\mathrm{Vs}(\mathrm{Z})$ rising by $27 \%$. Interestingly, we also observe that anisotropy for both $\mathrm{P}$ waves and S-waves decreases on initial application of pressure, but reaches a relatively low, stable value at pressures above about $30 \mathrm{MPa}$ (Figure 8). Since velocity anisotropy is heavily influenced by cracks, these observations further support the implication that the void space in COS contains a significant crack component which is effectively closed at hydrostatic pressures above about 30MPa. Since the initial porosity of COS is low (4.5\%), crack closure would also imply that the remaining pore space would be significantly less well connected. This interpretation is entirely consistent with the permeability data which show that approximately $80 \%$ of the total permeability reduction is achieved by the inferred crack closure pressure.

Again, BHS exhibits a contrasting response to the application of hydrostatic pressure. Although BHS has a much higher initial porosity than COS $(22.9 \%$ as against $4.4 \%$ ), the relative decrease in porosity is much lower at just over $3 \%$. This decrease is associated with increases of between $10.5 \%$ and $10.9 \%$ for all wave velocities (Figure 7). Most of the velocity increase occurs at pressures below $50 \mathrm{MPa}$, with the rate of increase slowing markedly above this value. Initial velocity anisotropies are rather low, and decreases to relatively stable values of $1-2 \%$ above about $10 \mathrm{MPa}$. Taken together, these data suggest a microstructure comprising a clean, well sorted matrix with a well-connected network of relatively equant pores and a low density of microcracks. This is in agreement with SEM observations as shown in Figure 1. 


\section{Summary and Conclusions}

Studies of the physical properties of rocks often focus on the measurement of a single parameter, and often under fixed conditions. In this study, we have expanded this to investigate multiple rock properties simultaneously at ambient and elevated pressures through the development of novel equipment and experimental techniques. Using the initial knowledge of pore space shape and orientation measured using pAMS and velocity anisotropy methods, we are able to better understand subsequent measurements conducted under hydrostatic pressure.

We conclude that both pAMS and velocity anisotropy methods can be used to characterize pore space anisotropy in rock without prior assumptions about principal directions [3]. This is particularly useful when no aligned fabric can be seen at the sample scale. The pAMS method is more efficient than the acoustic velocity technique, since it can produce a true 3D measurement from only a single core taken in any random direction. Both the pAMS and wave velocity measurements confirm that, for Crab Orchard sandstone, the fabric visible in hand specimen is representative of the anisotropy of the internal pore fabric. This fabric exerts a strong influence on fluid flow and its anisotropy. Although no such fabric is visible in samples of Bentheim sandstone, the measurements reveal that it also possesses an anisotropic fabric. However, in this case it does not appear to influence the fluid flow characteristics.

Under hydrostatic pressure, low aspect ratio cracks close more easily than high aspect ratio pores. The effect of pressure on acoustic wave velocity and permeability anisotropy of Crab Orchard sandstone therefore suggests that its microstructure contains a significant crack element which effectively closes at 40 to $50 \mathrm{MPa}$. In contrast, the results for Bentheim sandstone are more consistent with a microstructure dominated by equant pores. If the background mAMS is low, then pAMS directly measures the average anisotropic fabric of the connected pore space. It is therefore likely to be a good predictor of permeability anisotropy. However, the vast majority of measurements of anisotropy made at larger scale and in boreholes use acoustic or seismic methods. It is therefore important to correlate the two techniques so that 
detailed measurements made on core samples in the laboratory can be used to aid the interpretation of field scale data.

\section{Acknowledgements}

PMB was supported by the UK Natural Environment Research Council (NERC); award NER/S/A/2001/06507. Further support was provided by NERC grant GR9/03963 to PGM and ESP. Crab Orchard sandstone was kindly donated by Silvara Stone Co., Crossville, Tennessee. 


\section{References}

[1] Klimentos, T., 1991, The effects of porosity-permeability-clay content on the velocity of compressional waves. Geophysics, 56, 1930-1939.

[2] Bourbie, T., and Zinszner, B., 1985, Hydraulic and acoustic properties as a function of porosity in Fontainebleau Sandstone. J. Geophys. Res., 90, 1152411532.

[3] Benson, P.M., Meredith, P.G., and Platzman, E.S., Relating pore fabric geometry to acoustic and permeability geometry in Crab Orchard sandstone: A laboratory study using magnetic ferrofluid. Geophys. Res. Lett., 30(19), 1976, doi:10.1029/2003GL017929, 2003.

[4] Hornby, B.E., 1998, Experimental laboratory determination of the dynamic elastic properties of wet, drained shales. J. Geophys. Res., 103, 29945-29964

[5] Louis, C.D., David, C. and Robion, P., 2003, Comparison of the behaviour of undeformed sandstones in dry and wet conditions, Tectonophysics, 370 (1-4), 193-212.

[6] Tarling, D. H., and Hrouda, F., 1993, The Magnetic Anisotropy of Rocks. Chapman and Hall., London., pp 217.

[7] Pfleiderer, S., and Halls, H.C., 1990, Magnetic susceptibility anisotropy of rocks saturated with ferrofluid: a new method to study pore fabric? Phys. Earth \& Plan. Int., 65, 158-164.

[8] Pfleiderer, S., and Halls, H.C., 1994, Magnetic pore fabric analysis: a rapid method for estimating permeability anisotropy. Geophys. J. Int., 116, 39-45

[9] Rasolofosaon, P.N.J. and Zinszner, B.E, 2002, Comparison between permeability anisotropy and elastic anisotropy of reservoir rocks. Geophysics, 67, 230-240.

[10] Birch, F., 1960, The velocity of compressional waves in rocks to 10 kilobars, part 1, J. Geophys. Res., 65, 1083-1102

[11] Jones, C., and Meredith, P., 1998, An experimental study of elastic wave propagation anisotropy and permeability anisotropy in an illitic shale, Eurock 98, Proc. SPE/ISRM Rock Mechanics in Petroleum Engineering, Vol, 1, 307314.

[12] Nur, A., and Byerlee, J.D., 1971, An exact effective stress law for elastic deformation of rock with fluids, J. Geophys. Res., 76, 6414-6419 


\section{Tables}

Table 1. Anisotropy magnitude data for pAMS, P-wave anisotropy and S-wave anisotropy $(\mathrm{P}=(\max -\mathrm{min}) /$ mean $)$. Errors are calculated from the statistical deviation from 27 ellipsoids, and $\chi$ denotes susceptibility and $\mathrm{V}$ denotes the velocity for the rocks listed.

\begin{tabular}{|c|c|c|c|c|c|c|c|c|c|c|c|c|}
\hline \multirow[b]{2}{*}{$\begin{array}{l}\text { BHS } \\
\text { Value }(\chi / v)\end{array}$} & \multicolumn{4}{|c|}{ pAMS, $\chi\left(\times 10^{-6} \mathrm{SI}\right)$} & \multicolumn{4}{|c|}{$\mathrm{Vp}(\mathrm{m} / \mathrm{s})$} & \multicolumn{4}{|c|}{$\mathrm{Vs}(\mathrm{m} / \mathrm{s})$} \\
\hline & $\begin{array}{l}\text { Max. } \\
26503\end{array}$ & $\begin{array}{l}\text { Int. } \\
26388\end{array}$ & $\begin{array}{l}\text { Min. } \\
26153\end{array}$ & $\begin{array}{l}\mathrm{P}_{\mathrm{AMS}}, \% \\
1.5\end{array}$ & $\begin{array}{l}\text { Max. } \\
3461\end{array}$ & $\begin{array}{l}\text { Int. } \\
3392\end{array}$ & $\begin{array}{l}\text { Min. } \\
3302\end{array}$ & $\begin{array}{l}\mathrm{P}_{\mathrm{v}}, \% \\
4.7\end{array}$ & $\begin{array}{l}\text { Max. } \\
2089\end{array}$ & $\begin{array}{l}\text { Int. } \\
2074\end{array}$ & $\begin{array}{l}\text { Min. } \\
2028\end{array}$ & $\begin{array}{l}\mathrm{P}_{\mathrm{v}}, \% \\
3.0\end{array}$ \\
\hline Error & 16 & 24 & 27 & 0.2 & 4 & 4 & 5 & 0.2 & 4 & 2 & 5 & 0.4 \\
\hline $\begin{array}{l}\text { COS } \\
\text { Value }(\chi / v)\end{array}$ & 11384 & 11252 & 10964 & 3.8 & 3098 & 2954 & 2550 & 19.1 & 1854 & 1829 & 1766 & 7.6 \\
\hline Error & 15 & 23 & 26 & 0.3 & 12 & 13 & 10 & 0.4 & 10 & 7 & 5 & 0.3 \\
\hline
\end{tabular}


Table 2. Anisotropy directional data as calculated from fitting ellipsoids to pAMS, Pwave velocity and S-wave velocity data. Errors calculated using Fisher statistics (minimum direction) and Bingham statistics (maximum and intermediate values).

\begin{tabular}{ll|rrr|rrr|rrr} 
& & \multicolumn{4}{|c}{ pAMS } & \multicolumn{4}{c|}{ Vp } & \multicolumn{3}{c}{ Vs } \\
& & Max. & \multicolumn{1}{|c|}{ Int. } & Min. & Max. & Int. & Min. & Max. & Int. & Min. \\
\hline Bentheim & Trend & 225 & 134 & 45 & 192 & 283 & 81 & 153 & 68 & 322 \\
Sandstone & Plunge & 7 & 3 & 79 & 3 & 9 & 81 & 27 & 1 & 65 \\
& Error & - & - & 7 & 9 & 7 & 2 & 28 & 25 & 6 \\
\hline Crab Orchard & Trend & 29 & 121 & 281 & 351 & 82 & 249 & 87 & 84 & 269 \\
Sandstone & Plunge & 5 & 16 & 73 & 2 & 7 & 83 & 31 & 22 & 64 \\
& Error & 11 & 9 & 3 & 2 & 3 & 2 & - & - & 5
\end{tabular}




\section{Figure Captions}

Figure 1. Scanning electron micrographs of Bentheim sandstone (left) and Crab Orchard sandstone (right).

Figure 2. Core orientations used in this study. Note the position of the 'zero degrees' starting position.

Figure 3. Schematic diagram of permeameter/volumometer used for simultaneous porosity, permeability and acoustic wave velocity measurements.

Figure 4. Detail of permeameter/volumometer sample assembly showing key components.

Figure 5. P-wave and S-wave velocity variation with radial angle for BHS (left) and $\operatorname{COS}$ (right). Plots labels refer to the core axis, for example ' $\mathrm{X}$ ' refers to radial measurements made in the YZ plane (fig 1).

Figure 6. pAMS, Vp and Vs (left to right) anisotropy directional data stereonets for BHS (top) and COS (bottom). Circles denote minimum principal directions, triangles intermediate directions, and squares denote maximum principal directions.

Figure 7. Permeability, P-wave velocity and S-wave velocity comparison between BHS (left) and COS (right). In the legend, $\mathrm{Vp}(\mathrm{X}) / \mathrm{Vp}(\mathrm{Y})$ denotes the average $\mathrm{P}$ wave velocity over both the $\mathrm{X}$ and $\mathrm{Y}$ directions, and $\mathrm{Vp}(\mathrm{Z})$ denotes the $\mathrm{P}$-wave velocity in the $\mathrm{Z}$ direction.

Figure 8. Comparison of acoustic velocity anisotropy for BHS (left) and COS (right).

Figure 9. Permeability variation with effective pressure and key coring direction with respect to pAMS and acoustic velocity anisotropy for BHS (left) and COS (right).

Figure 10. Comparison of permeability anisotropy between BHS and COS. 
Figures

Figure 1.
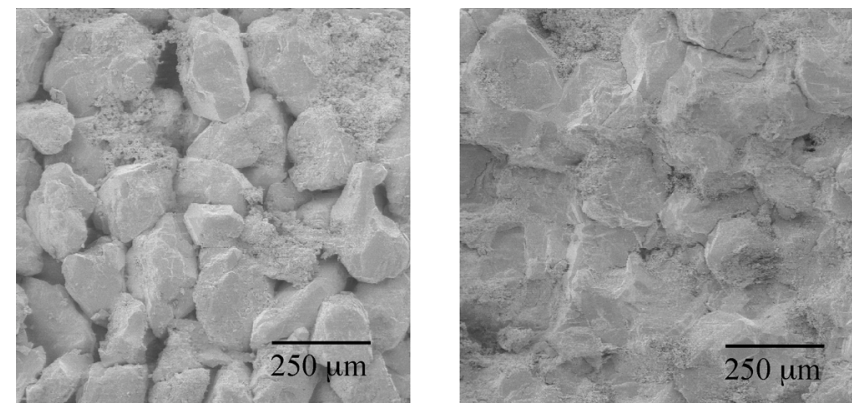
Figure 2.

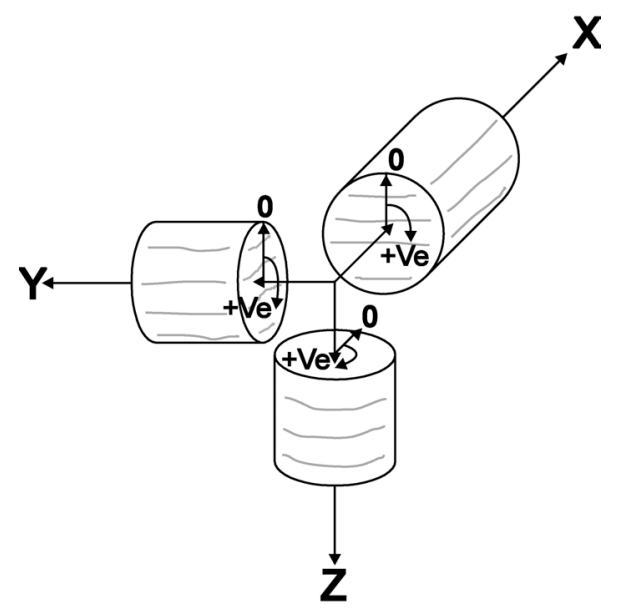


Figure 3.

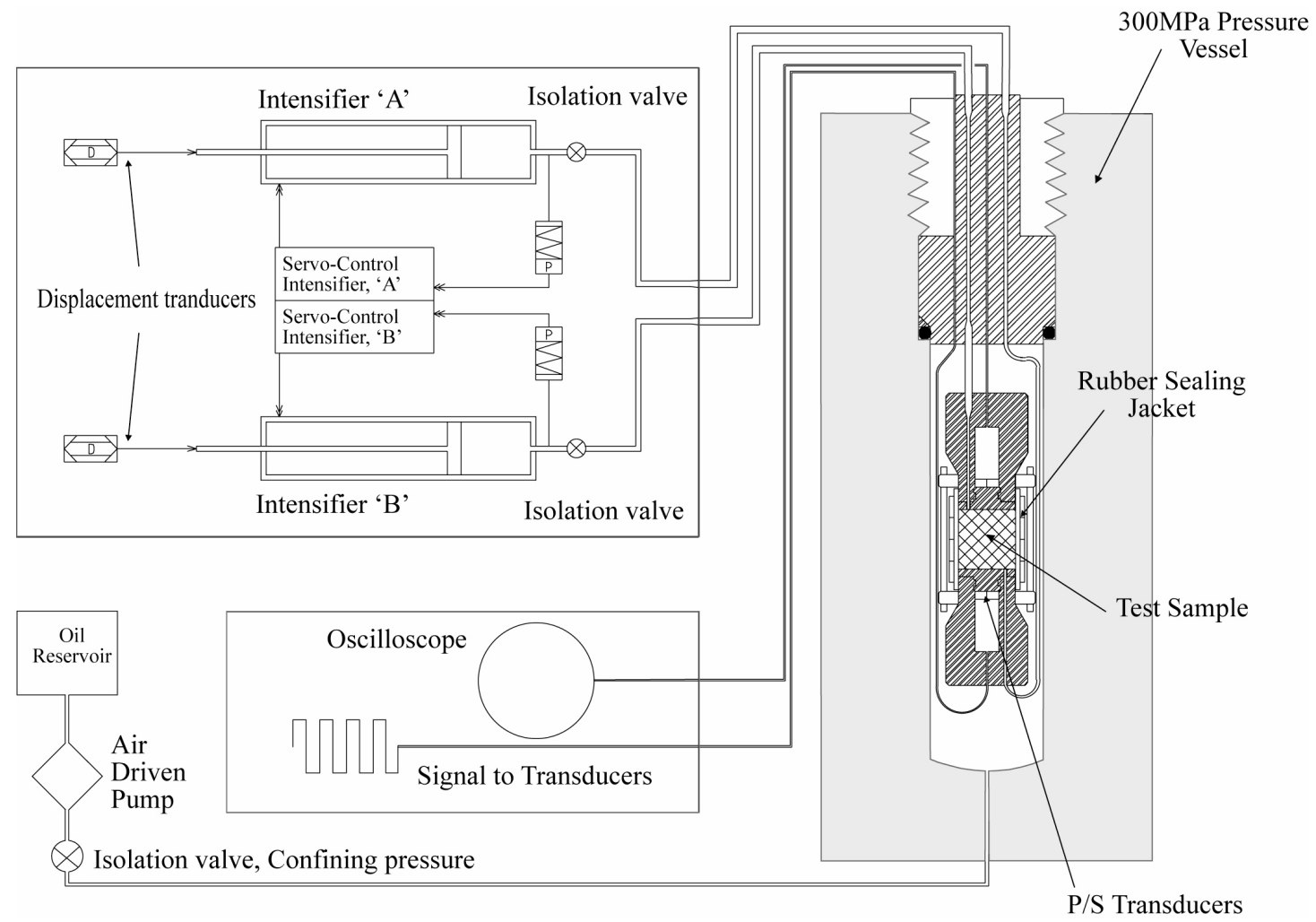


Figure 4.

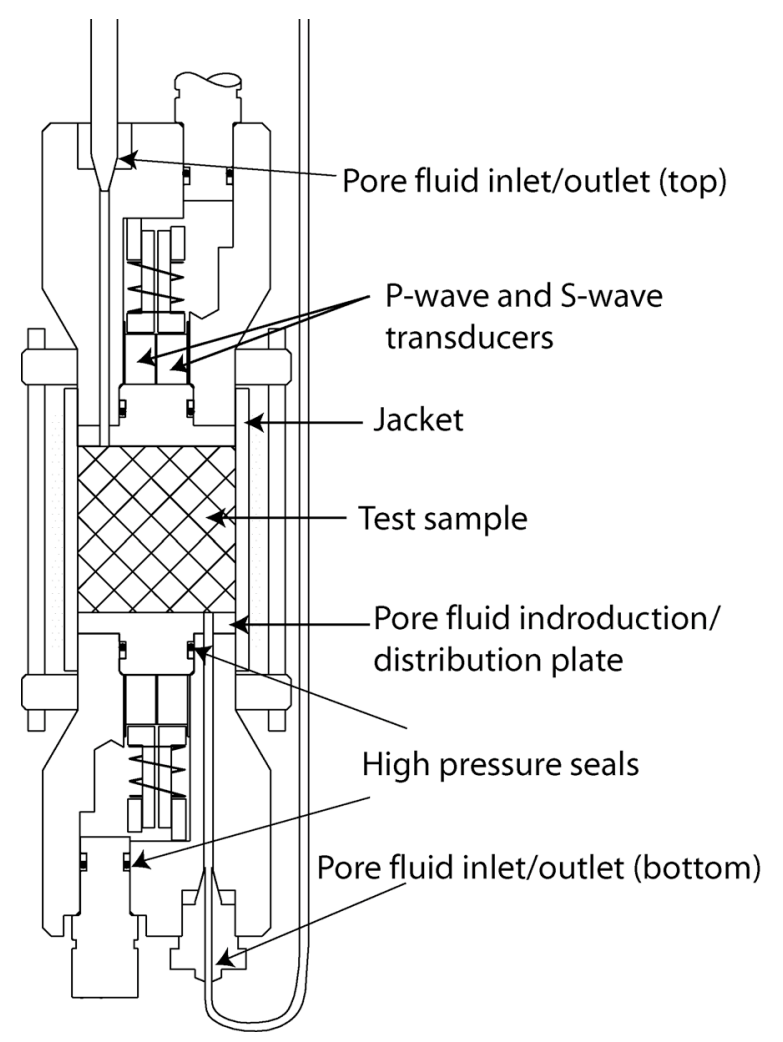


Figure 5.
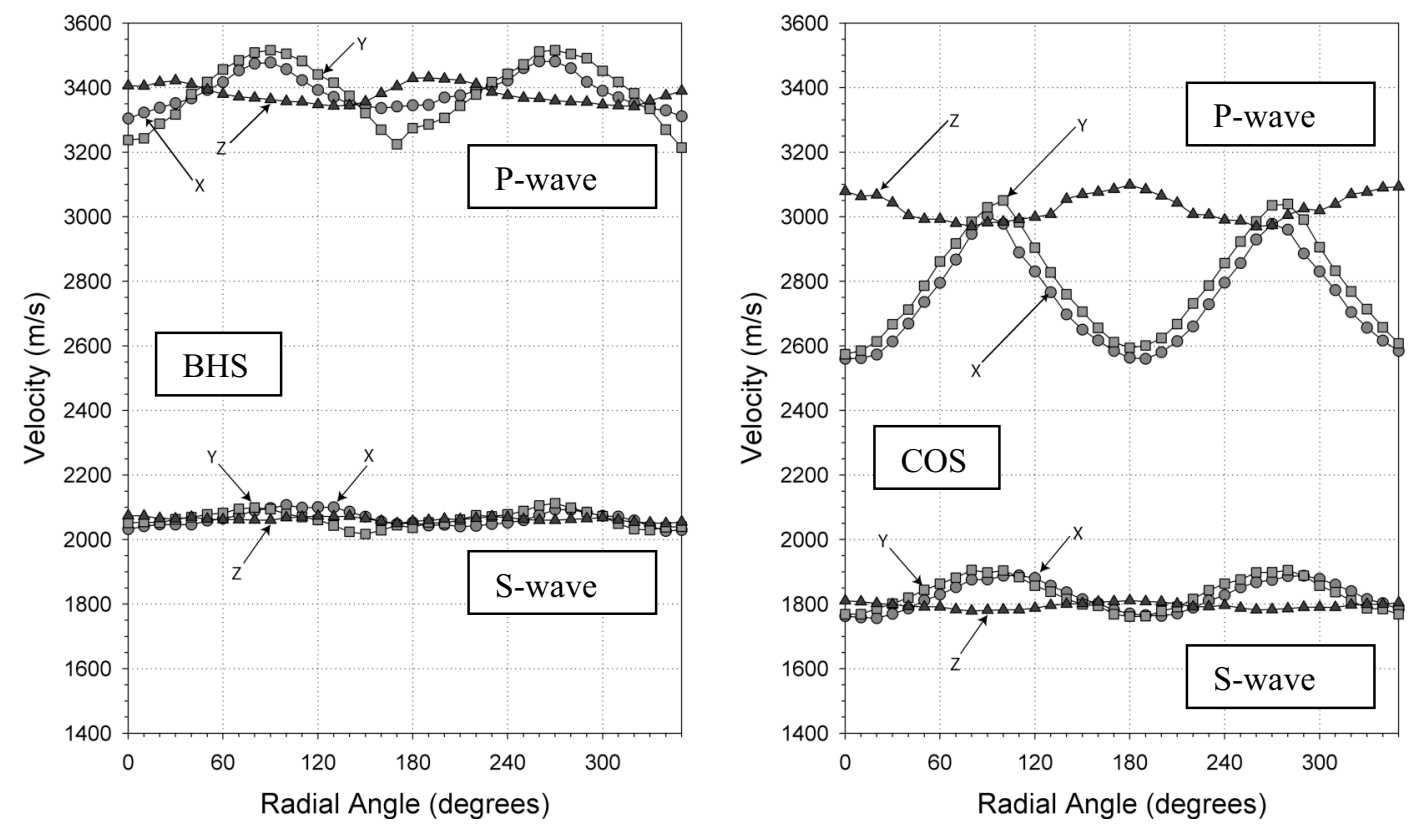
Figure 6 .
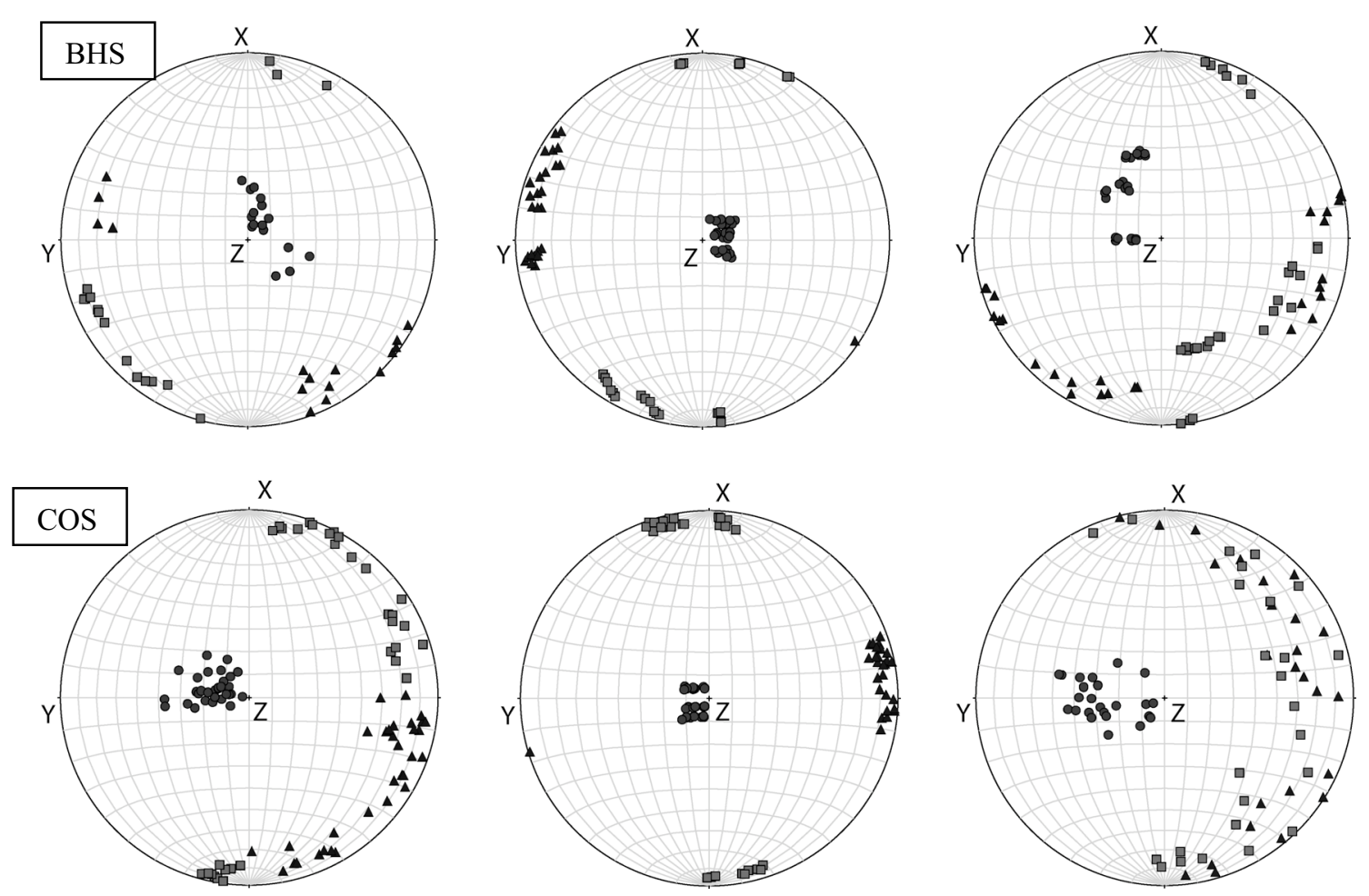
Figure 7.
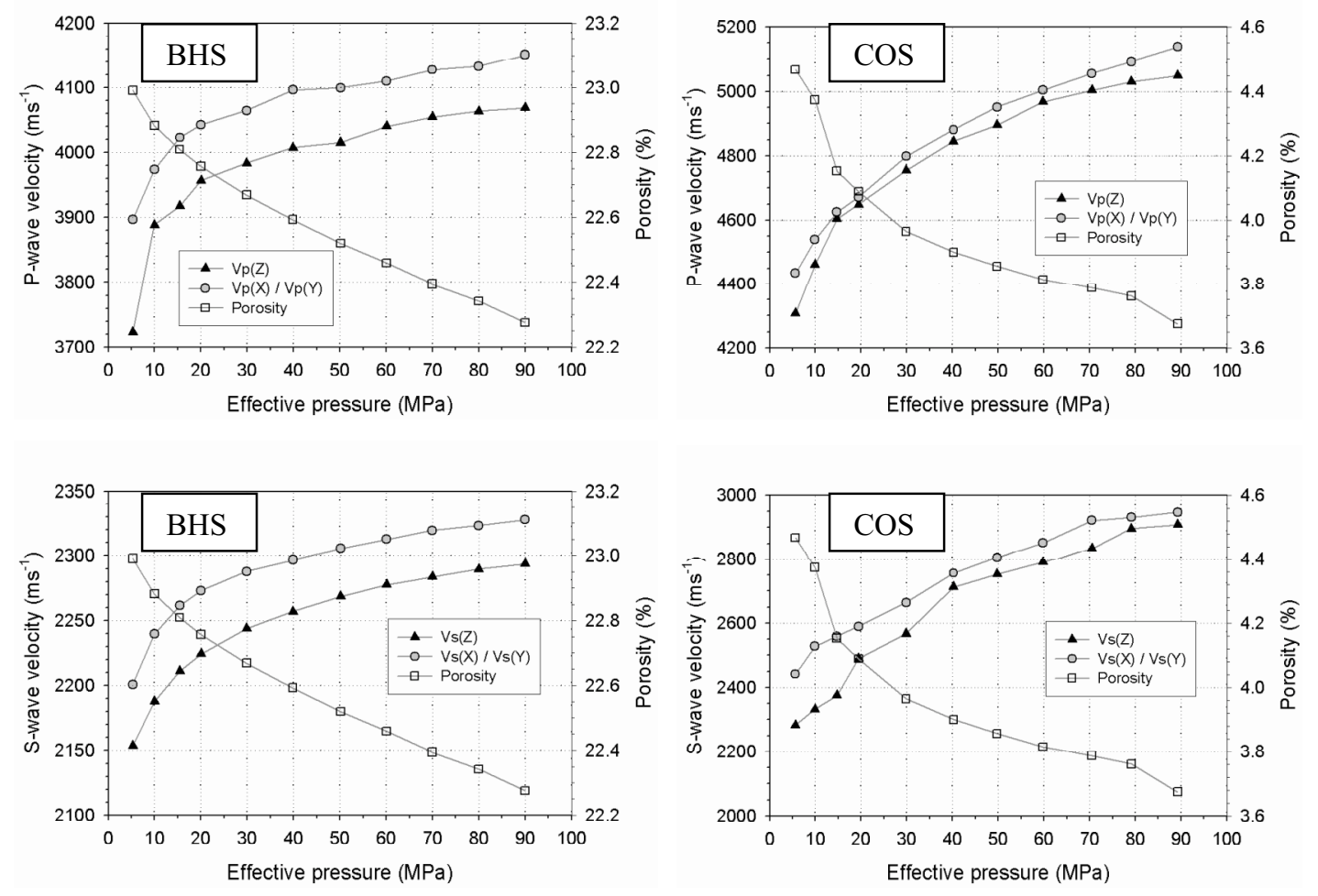
Figure 8.
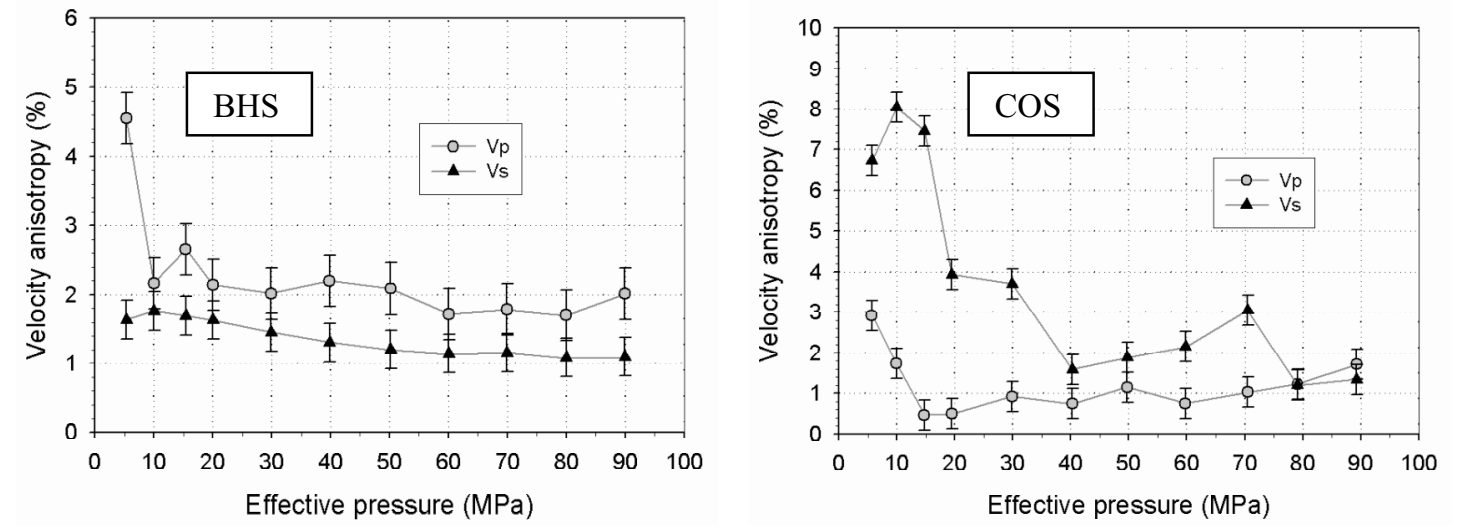
Figure 9.
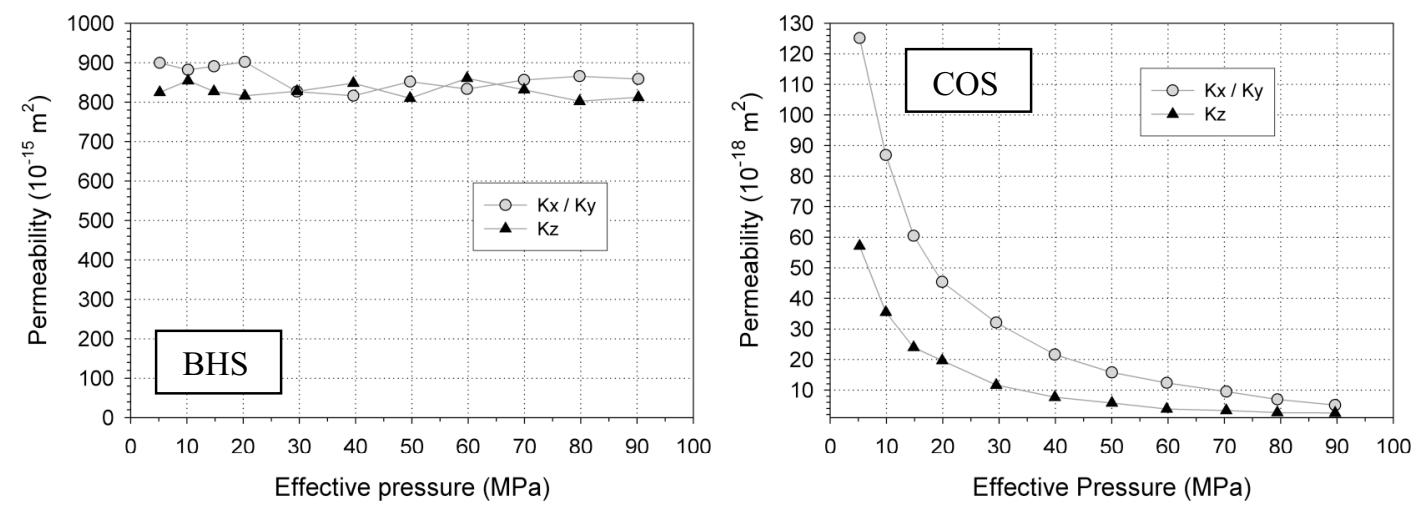
Figure 10.

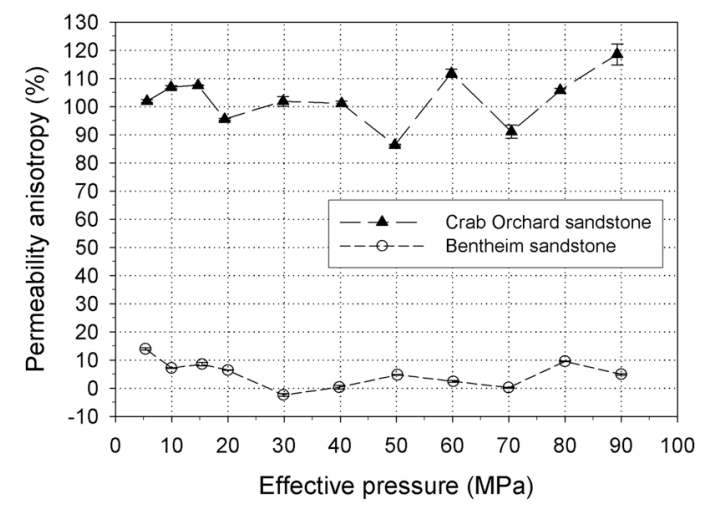

